01-industrial 27-Kleeberg:REVISTA INGENIERIA 27ł $01 / 2011$ 11:38 PEgina 10

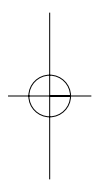




\title{
Aplicación de las técnicas de muestreo en los negocios y la industria
}

\author{
Fernando Kleeberg Hidalgo, Julio César Ramos Ramírez
}

Ingeniería Industrial n. 27, 2009, ISSN 1025-9929, pp.11-40

RESUMEN: Este trabajo resume los aspectos relevantes del muestreo como disciplina que trata con el conjunto de técnicas para obtener una muestra, y busca orientar las actividades de los investigadores que requieren recolectar datos por muestreo. Se organizó a partir de la definición de términos técnicos y se continuó con la clasificación de las técnicas del muestreo según su selección y complejidad. Sobre este último punto se destacan las técnicas del muestreo complejo para el diseño de encuestas, que se ha convertido en la herramienta más común y poderosa para obtener información de poblaciones muy grandes a un costo relativamente bajo. Por consiguiente, la utilidad del muestreo se da en toda organización que necesite información oportuna, confiable y con el costo más bajo posible. El artículo concluye con una revisión de los aspectos más relevantes del muestreo de aceptación utilizado en los procesos de control de calidad.

Palabras clave: muestreo / tipos de muestreo / muestreo de aceptación

\section{Sampling techniques in business and industry}

ABSTRACT: This document makes a summary of relevant aspects of sampling as a discipline that deals with the set of techniques to make or obtain a sample, and seeks to guide the activities of those researchers who need to collect data for their research sample. The work was organized around the definition of technical terms used in sampling, according to the classification of the techniques of sampling from a selection from the viewpoint of complexity of sampling. This last point highlights the complex sampling techniques for the design of surveys, which now has become the most common and powerful tool to obtain information about large populations at relatively low cost. Therefore the usefulness of sampling is given in any organization that needs information timely, reliable at a low cost. The document concludes with a review of the most relevant aspects of the acceptance sampling, used in quality control processes.

Keywords: sampling / types of sampling / sampling acceptance 


\section{INTRODUCCIÓN}

David Ruiz nos explica que la estadística es mucho más que solo números apilados y gráficas bonitas. ${ }^{1}$ Es una ciencia tan antigua como la escritura, y es por sí misma auxiliar de todas las demás ciencias. Los mercados, la medicina, la ingeniería, los gobiernos, etcétera, se cuentan entre sus más destacados clientes.

El objetivo de la estadística es hacer inferencias acerca de una población con base en la información contenida en una muestra. Este mismo objetivo motiva el estudio del problema de muestreo. Por consiguiente, el muestreo se define como la disciplina que trata con el conjunto de técnicas para tomar u obtener una muestra. Si las técnicas se basan en las leyes de la probabilidad se denomina "muestreo probabilístico", pero si las técnicas no consideran los principios de probabilidad se le llama "muestreo no probabilístico".

Los estudios por muestreo son empleados por el ingeniero industrial que desea obtener información para controlar la calidad de la producción de una fábrica; los ecólogos, para construir mapas de comunidades de plantas; el silvicultor, que requiere conocer el rendimiento de madera de un bosque, y el gerente de un servicio de evaluación (rating), que desea determinar la popularidad de programas de televisión entre los espectadores. Asimismo, se usan para pronosticar la recolección de la cosecha, identificar las condiciones sociales y económicas prevalecientes, como desempleo, cuidado de la salud, inflación, y para examinar las actitudes de los ciudadanos hacia una legislación propuesta. Un barómetro de opiniones públicas, tan importante para una democracia, se proporciona prontamente por los estimativos basados en una muestra de la reacción pública a los efectos de tales eventos como una nueva regulación salarial, un cambio mayor en políticas de comercio o las acciones de líderes mundiales.

Usualmente, estas investigaciones utilizan técnicas cuantitativas de recopilación de información, que permiten su extrapolación (es posible la inferencia), pues procede de muestras obtenidas por procedimientos estadísticos. Es frecuente que las investigaciones se desarrollen con fines de cuantificación. Antes de las elecciones, las empre-

1 Ruiz Muñoz, D. Manual de estadística, 2008.

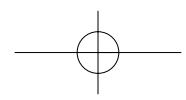


sas de investigación de mercados estiman los votos que conseguirán los diferentes candidatos, por ejemplo. La principal técnica cuantitativa de recopilación de información es la encuesta personal.

\section{TÉRMINOS TÉCNICOS EN EL MUESTREO}

Para facilitar una comprensión de la naturaleza del estudio muestral, que es común a muchos campos de aplicación, se empezará con definiciones para continuar con aplicaciones del muestreo según los campos de la ingeniería; por último, se presentarán algunos ejemplos aplicando programas de cálculos rápidos.

- Población. Una población es un conjunto de individuos u objetos (unidades de muestreo o de estudio) que poseen la característica que se desea estudiar. La población ideal que se pretende estudiar se denomina población objetivo. El grupo que en realidad podemos estudiar se denomina población de estudio.

- Muestra.- Es un subconjunto extraído de la población, por tanto es el conjunto de mediciones que han sido realmente recolectadas.

- Muestra aleatoria. Es una colección de variables aleatorias independientes idénticamente distribuidas. Se dice que una muestra aleatoria es "representativa" de la población debido a que considera que cada elemento de la población ha tenido la misma oportunidad de formar parte de la muestra. Las conclusiones basadas en una muestra aleatoria son generalizables a la población de la muestra.

- Unidad de análisis. Es la unidad elemental o de observación que corresponde a la entidad que va a ser objeto específico de estudio en una medición y se refiere al qué o quién es objeto de interés en una investigación; por ejemplo, una familia u hogar, un trabajador, un estudiante, un consumidor, etcétera.

- Unidad de muestreo. Corresponde a la entidad básica mediante la cual se accederá a la unidad de análisis. En algunos casos, ambas unidades se corresponden. La unidad de muestreo debe ser observable, identificable y ubicable; por ejemplo, una vivienda, una empresa, un colegio, un centro comercial, etcétera.

- Unidad informante. Corresponde a la entidad que reporta la información sobre la unidad de análisis observada; por ejemplo, el jefe de familia, el trabajador, el estudiante, el consumidor, etcétera. 
- Marco muestral. Es el colectivo de todas las unidades muestrales existentes en la población de estudio. Se organiza y consolida en una base de datos tabular o gráfica, que permitirá diseñar, clasificar, seleccionar, identificar y ubicar las unidades de la muestra. Puede ser de dos tipos:

- Marco muestral de lista. Cuando las unidades de muestreo del marco se constituyen en un listado. Ejemplo: listado de agentes, listado de estudiantes, listado de empresas, etcétera.

- Marco muestral de áreas. Cuando las unidades de muestreo del marco son áreas de terreno usualmente llamados segmentos (segmentos de área) y dentro de ella suelen haber varias unidades de información. Las unidades se ubican a través de un sistema de coordenadas y se utilizan mapas y listados; por ejemplo, el marco de parcelas catastradas, el marco digital de manzanas de viviendas de Lima metropolitana, etcétera.

- Variable. Es la característica que se desea estudiar en las unidades de observación y pueden ser de dos tipos: cuantitativas (discretas y continuas) y cualitativas (nominal u ordinal).

- Dato. Es un valor particular que toma la variable de interés en una unidad de observación. El conjunto de datos obtenido con $\mathrm{p}$ variables y $\mathrm{n}$ unidades de observación se denomina matriz de datos.

- Parámetro. Es un valor que caracteriza a una población. El valor del parámetro es constante y por lo general desconocido. Los parámetros usuales para variables cuantitativas son la media poblacional, la total poblacional y la desviación estándar poblacional; para las variables cualitativas suele utilizarse la proporción o porcentaje poblacional.

- Peso muestral. El peso muestral, también denominado factor de expansión, es el valor numérico calculado para cada elemento de una muestra probabilística. Cada valor representa, aproximadamente, las veces que un elemento de la muestra se repite en la población. Estos valores se calculan a partir de las probabilidades de selección de la muestra diseñada para una encuesta.

- Error muestral o error de muestreo. Es la diferencia entre el resultado obtenido de una muestra (un estadístico) y el resultado que deberíamos haber obtenido de la población (el parámetro correspondiente). Mientras más pequeño el error muestral mayor es la precisión de la estimación. Los indicadores del error muestral son los siguientes: 
- Error estándar del estimador (EE), definido como la raíz cuadrada de la varianza del estimador.

- Error relativo de muestreo (ERM), definido como el coeficiente de variación del estimador. Este indicador es el más importante en las encuestas por muestreo y se interpreta como:

Si ERM $\leq 5 \%$, la estimación es "muy buena".

Si 5\% < ERM£10\%, la estimación es "buena".

Si 10\% < ERM£15\%, la estimación es "aceptable".

Si 15\% < ERM£25\%, la estimación es "solo referencial".

- Error ajeno al muestreo. Son los errores que se introducen de manera imperceptible a la encuesta y los más difíciles de controlar. Desafortunadamente, estos errores no se pueden medir con facilidad, y aumentan a medida que aumenta el tamaño de la muestra. Los tipos de errores no muestrales que suelen presentarse son:

- Definición equivocada del problema.

- Definición defectuosa de la población.

- Marco imperfecto o desactualizado.

- La No respuesta.

- El sesgo de respuesta.

- Diseño pobre del instrumento de medición.

Sin embargo, los errores de no muestreo pueden ser controlados mediante una atención cuidadosa en todas las etapas de la encuesta.

\section{LAS TÉCNICAS DE MUESTREO}

\subsection{Clasificación de las técnicas de muestreo}

Las técnicas de muestreo se pueden clasificar por su forma de selección y por su nivel de complejidad. Una clasificación de las técnicas de muestre según la forma de selección y el nivel de complejidad se presenta en al cuadro 1. 
Fernando Kleeberg, Julio César Ramos

\section{Cuadro 1}

Clasificación de las técnicas de muestreo

\begin{tabular}{lcccc}
\hline & \multicolumn{2}{c}{ Forma de selección } & \multicolumn{2}{c}{ Nivel de complejidad } \\
\hline Técnica & $\begin{array}{c}\text { Muestreo } \\
\text { probabilístico }\end{array}$ & $\begin{array}{c}\text { Muestreo no } \\
\text { probabilístico }\end{array}$ & $\begin{array}{c}\text { Muestreo } \\
\text { simple }\end{array}$ & $\begin{array}{c}\text { Muestreo } \\
\text { complejo }\end{array}$ \\
\hline Muestreo aleatorio simple & $\mathrm{X}$ & $\mathrm{X}$ & \\
Muestreo sistemático & $\mathrm{X}$ & $\mathrm{X}$ & \\
Muestreo aleatorio estratificado & $\mathrm{X}$ & $\mathrm{X}$ & \\
Muestreo por conglomerados & $\mathrm{X}$ & & $\mathrm{X}$ \\
sin submuestreo & $\mathrm{X}$ & & & $\mathrm{X}$ \\
Muestreo por conglomerados & $\mathrm{X}$ & & $\mathrm{X}$ \\
con submuestreo & $\mathrm{X}$ & $\mathrm{X}$ & $\mathrm{X}$ \\
Muestreo multietápico & & $\mathrm{X}$ & $\mathrm{X}$ & \\
Muestreo por fases & & $\mathrm{X}$ & $\mathrm{X}$ & \\
Muestreo por conveniencia & & $\mathrm{X}$ & $\mathrm{X}$ & \\
Muestreo por juicio & & $\mathrm{X}$ & \\
Muestreo "bola de nieve" & & & & \\
Muestreo por cuotas & & & &
\end{tabular}

Fuente: Bhattacharyya, Gouri K. y Richard A. Johnson. Statistical concepts and methods, 1977

Cuando la técnica de muestreo asigna a cada unidad de la población alguna probabilidad (diferente de cero) de ser seleccionada; se le denomina muestreo probabilístico. Por otro lado, se le llama muestreo no probabilístico cuando no se asigna probabilidad de selección alguna a las unidades de la población; es decir, no se conoce la probabilidad y por tanto no se puede extrapolar o realizar inferencia estadística.

En el cuadro 2 se presentan las fortalezas y debilidades del muestreo probabilístico y no probabilístico: 
Cuadro 2

Técnicas de muestreo probabilístico

\begin{tabular}{ll}
\hline Fortalezas & Debilidades \\
\hline $\begin{array}{ll}\text { Es aceptado con facilidad } & \text { Requiere de costosos marcos de muestreo } \\
\text { Asegura representatividad } & \text { Requiere de trabajos de campo costosos } \\
\text { Asegura muestras insesgadas } & \\
\text { Resultados son generalizables. } & \\
\text { Permite calcular error de muestreo. } & \\
\text { Permite hacer inferencias estadísticas } & \\
\text { Asegura el anonimato del entrevistado } & \\
\text { Personal de campo no especializado } & \\
\text { Puede ser supervisada } & \\
\text { Probabilidad conocida de elegir a la unidad } & \\
\text { como parte de la muestra. }\end{array}$ \\
\hline
\end{tabular}

Técnicas de muestreo no probabilístico

\begin{tabular}{ll}
\hline Fortalezas & Debilidades \\
\hline Bajo costo y requiere menor tiempo & No permite calcular errores de muestreo \\
Elige solo las unidades requeridas & Sus resultados no son generalizables \\
Permite encontrar información inesperada & No permite calcular errores de muestreo \\
Diseño y cobertura flexible e informal & Sus resultados no son generalizables \\
& Pueden ser rechazadas por los usuarios \\
& Puede haber sesgo en la selección \\
& No permite hacer inferencia estadística \\
& Requiere de personal muy especializado \\
& No asegura el anonimato del entrevistado \\
& La supervisión es difícil de realizar \\
& Probabilidad no conocida de elegir a la \\
& unidad como parte de la muestra. \\
\hline
\end{tabular}

Fuente: Rubio, Arturo. "Técnicas de muestreo". Asignatura en la Maestría de Estadística Aplicada, Universidad Nacional Agraria La Molina, 2005.

\subsection{Técnicas de muestreo probabilístico}

\subsubsection{Muestreo elemental directo}

Cuando las unidades de la población se encuentran identificadas en un marco muestral de lista, el muestreo probabilístico directo de elementos es el más adecuado para seleccionar la muestra. Las técnicas que se pueden aplicar en el muestreo directo de elementos son de muestreo aleatorio simple, muestreo sistemático y muestreo aleatorio estratificado. Estas técnicas se caracterizan por asignar igual proba- 
bilidad a las diferentes unidades de la población, generando pesos muestrales o factores de expansión constantes. A este tipo de diseños muestrales también se les denomina diseños autoponderados.

Para el tratamiento y el análisis de una muestra elemental directa existen una diversidad de software estadístico, como Minitab, Systat, Statistica, etcétera.

- Muestreo aleatorio simple (MAS).- Se usa cuando a cada elemento de la población se le quiere dar la misma oportunidad de ser elegido en la muestra. Se puede realizar partiendo de listas de individuos de la población y eligiendo individuos aleatoriamente con un ordenador. Normalmente, su aplicación tiene un costo elevado. En el gráfico 1 se presenta el muestreo aleatorio simple:

\section{Gráfico 1}

Muestreo aleatorio simple

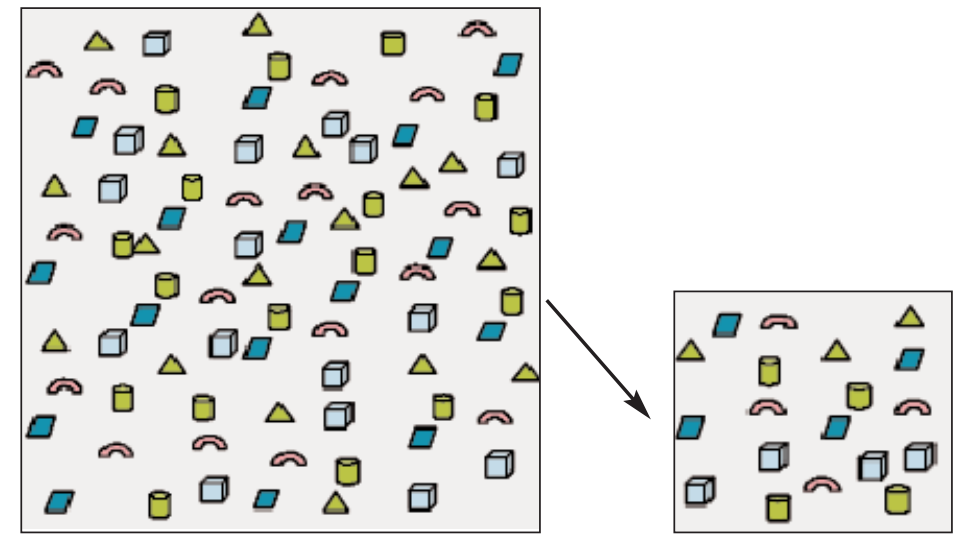

Fuente: Casal, J. y E. Mateu. "Tipos de muestreo". Revista de Epidemiología y Medicina Preventiva 1, 2003.

- Muestreo sistemático.- Es un tipo de muestreo aleatorio que se usa cuando los datos de la población de estudio están ordenados en forma numérica. La primera observación es elegida al azar entre los primeros elementos de la población y las siguientes observaciones son elegidas guardando la misma distancia entre sí. Hay que tener cuidado con las muestras donde existen periodicidades, porque pueden resultar muestras sesgadas. En el gráfico 2 se observa el muestreo sistemático: 
Gráfico 2

Muestreo sistemático

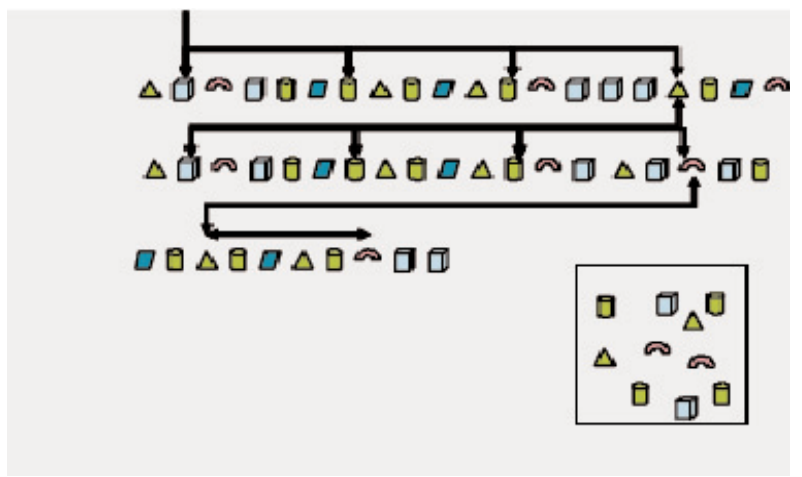

Fuente: Ibídem.

- Muestreo aleatorio estratificado.- Es un tipo de muestreo aleatorio que se usa cuando se conoce de antemano que la población está dividida en estratos (variable, subpoblaciones, etcétera), que son equivalentes a categorías y que, por lo general, no son de igual tamaño. Luego, de cada estrato se saca una muestra aleatoria, usualmente proporcional al tamaño del estrato. Se realiza un MAS de los individuos de cada uno de los estratos. Al extrapolar los resultados a la población, hay que tener en cuenta el tamaño relativo del estrato con respecto al total de la población. En el gráfico 3 se presenta el muestreo estratificado:

Gráfico 3

Muestreo estratificado.

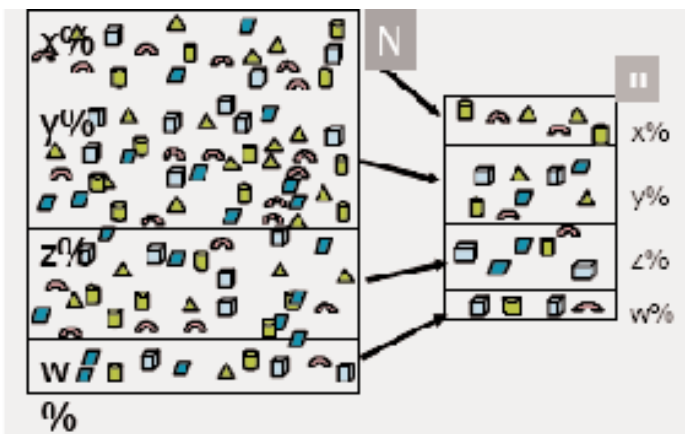

Fuente: Ibídem. 


\subsubsection{Muestreo complejo}

Cuando las unidades de la población no se encuentran identificadas en un marco muestral de lista o la elaboración del listado es demasiado costoso y complejo de elaborar por tratarse de una población muy grande, el muestreo probabilístico más adecuado para seleccionar la muestra resulta ser el muestreo por conglomerados (con o sin submuestreo) o muestreo multietápico. Este tipo de muestreo es denominado muestreo complejo, por las siguientes razones:

- Utiliza marcos muestrales de unidades grandes o grandes conglomerados de elementos que son seleccionados con probabilidades desiguales proporcionales a sus tamaños.

- La muestra se selecciona en varias etapas de muestreo, generando submuestras dentro de cada conglomerado seleccionado.

- El proceso de estimación de los parámetros es complicado y requiere el uso de pesos muestrales diferentes en las unidades finales de muestreo.

- La estimación del error muestral es complejo y requiere métodos especiales para la estimación de varianzas.

- Los tamaños de muestra se calculan considerando el grado de complejidad del diseño muestral.

El uso de diseños complejos de muestreo está en concordancia con la complejidad de la población de estudio, lo cual en cierta medida abarata los costos de recolección de información. En general, la complejidad en el diseño muestral está muy relacionada con la complejidad del procedimiento de estimación. Sin embargo, las técnicas estadísticas empleadas en el análisis de encuestas en su gran mayoría no toman en cuenta la complejidad del diseño muestral o la estructura de la población. Esto es una consecuencia que parte del supuesto común de que la muestra es independiente e idénticamente distribuida (iid) de alguna población.

Por lo tanto, para el tratamiento y análisis de una muestra compleja no es posible el uso de los programas estadísticos clásicos, como Minitab o Systat (que suponen iid); se requiere software especializado como el SPSS (módulo de "muestras complejas") o el STATA (módulo de "análisis de datos de encuestas"), los cuales utilizan pesos muestrales para el proceso de estimación y expansión. 


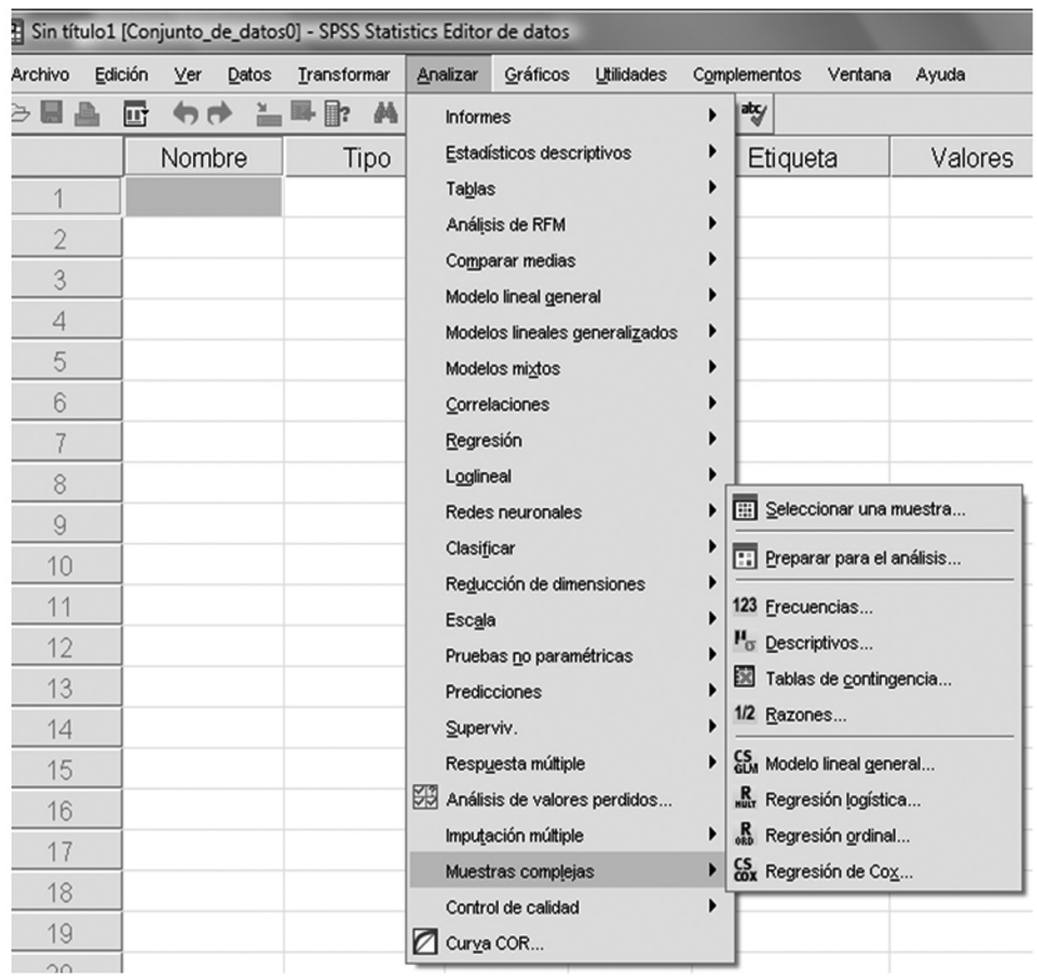

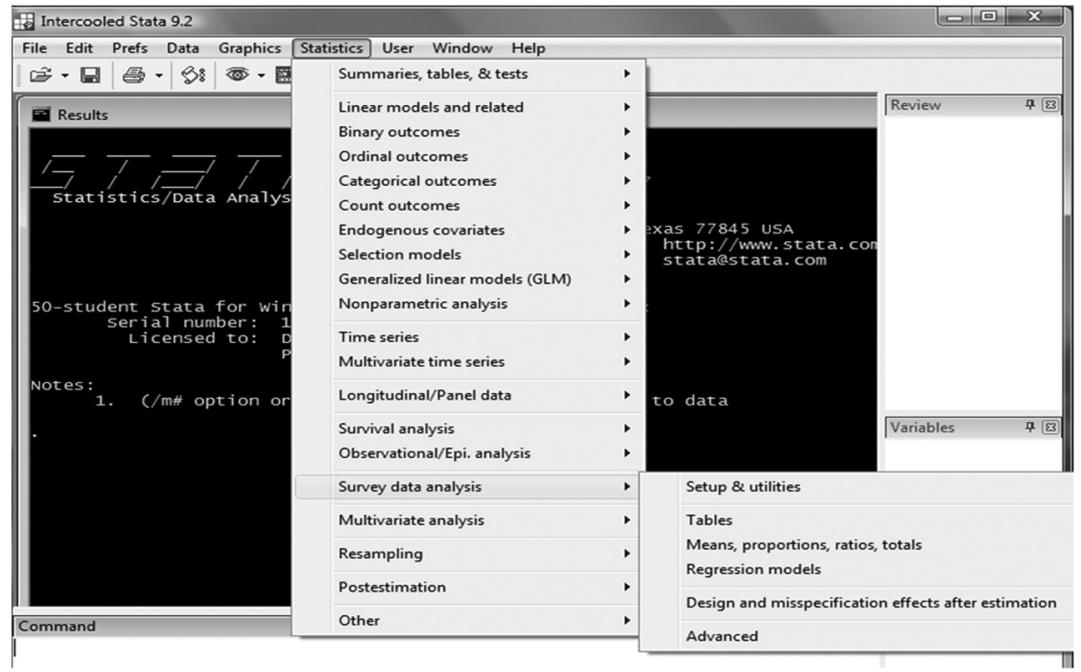


Los diseños muestrales complejos comúnmente combinan conglomeración y estratificación con probabilidad de selección desigual. Por ejemplo: un diseño muestral por conglomerados, estratificado, desproporcionado (por área metropolitana, manzana o vivienda), donde se entrevista a un adulto por hogar y ocurre la no respuesta. En este caso, es necesario el cálculo de pesos muestrales ajustados por la no respuesta para cada elemento de la muestra.

Los pesos muestrales eliminan el sesgo de estadísticos lineales (totales, medias, proporciones, etcétera), y reducen el sesgo de la componente no lineal en estadísticos no lineales (razones, coeficientes de correlación, parámetros de regresión logística, etcétera). Por lo tanto, si una muestra compleja es tratada como una muestra iid (como una m.a.s) y se utilizan estimadores de varianza estándar, se estará subestimando la varianza teórica del estimador. Esto significa que los intervalos de confianza que se generen, serán más angostos de lo que realmente son.

El muestreo por conglomerados con o sin submuestreo es la técnica que se utiliza en el muestreo complejo; se aplica cuando es difícil tener una lista de todos los individuos que forman parte de la población de estudio, pero sin embargo se sabe que se encuentran agrupados naturalmente en grupos. Se realiza cuando se eligen varios de esos grupos al azar, y ya elegidos algunos se puede estudiar a todos los individuos de los grupos seleccionados o bien seguir aplicando dentro de ellos más muestreos por grupos, por estratos, aleatorios simples. La eficiencia del muestreo por conglomerados exige heterogeneidad entre elementos del mismo conglomerado y homogeneidad entre elementos de diferentes conglomerados. Entonces, si los elementos de un mismo conglomerado son muy diferentes entre sí una muestra que contenga pocos conglomerados grandes puede producir una buena estimación del parámetro de interés. Raj dice que "un conglomerado es una especie de miniatura de la población". ${ }^{2}$

$\mathrm{Al}$ extrapolar los resultados a la población, hay que tener en cuenta el tamaño relativo de unos grupos con respecto a otros. En el gráfico 4 se presenta el muestreo por grupos o conglomerados:

2 Raj, D. Teoría del muestreo, 1984.

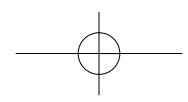




\section{Gráfico 4}

Muestreo por grupos o conglomerados

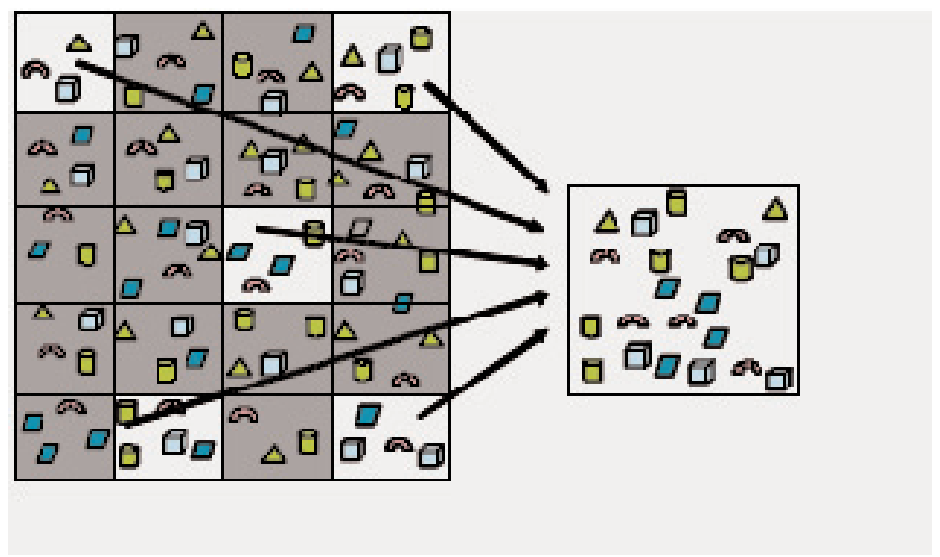

Fuente: Casal, J. y E. Mateu. Op. cit.

\subsection{Técnicas de muestreo no probabilístico}

- Muestras de conveniencia o accidentales.- Están constituidas por elementos que voluntariamente acceden a ser observados y medidos, llegan al estudio exploratorio en forma casual y su elección dependerá de circunstancias fortuitas.

- Muestras de expertos.- Están formadas por elementos integrantes de grupos determinados, dentro de los cuales si se eligen entre 8 a 10 sujetos típicos será posible identificar patrones de comportamiento específicos a través de sesiones en las que un facilitador motivará la participación individual con el fin de que expresen sus actitudes, preferencias y expectativas respecto a determinados productos o servicios

- Muestras de "bola de nieve".- Se elige una pequeña muestra inicial y a cada uno de los individuos seleccionados se le pide que sugieran a otros posibles individuos que podrían ser incluidos en la muestra, de acuerdo con requisitos previamente establecidos. También se le conoce como muestra en cadena o por redes, pues se identifica a unidades que llevarán a otras unidades que están íntimamente relacionadas y por tanto permitirán analizar la cadena. 
- Muestreos por cuotas.- Las unidades de análisis observadas, medidas o entrevistadas son elegidas completando cuotas establecidas con base en la proporción o significación que tiene dentro de la población. Finalmente, las unidades serán identificadas y seleccionadas de acuerdo con el juicio del investigador.

\section{EL PROBLEMA DEL TAMAÑO DE LA MUESTRA}

Se definen tres expresiones técnicas sobre los tamaños de muestra: el tamaño esperado de muestra, el tamaño planeado de muestra y el tamaño efectivo de muestra.

El tamaño esperado de muestra (TEM) resume solamente consideraciones estadísticas, de acuerdo con las exigencias sobre precisión y confiabilidad. Es el tamaño muestral necesario para lograr estimaciones de valores poblacionales con especificaciones de confiabilidad y precisión.

El tamaño planeado de muestra (TPM) incluye la provisión necesaria para la No Respuesta, lo que es de especial importancia cuando no se admiten sustituciones que siempre afectan al diseño muestral. Es el tamaño muestral necesario para satisfacer el tamaño esperado de muestra considerando la No Respuesta.

\section{TPM = TEM / Tasa Anticipada de Respuesta}

Por último, el tamaño final de muestra se refiere al número de entrevistas o cuestionarios que se han aceptado como válidos y que corresponden a la muestra. Es el número de unidades muestrales que finalmente son observadas (o entrevistadas) de acuerdo con las reglas y procedimientos de campo definidos para la encuesta.

\subsection{Tamaño de muestra en muestreo directo simple}

Cuando se está realizando el muestreo directo de elementos, por ejemplo m.a.s o muestreo sistemático, para estimar promedios el TEM se calcula a través de la siguiente fórmula:

$$
\mathrm{n}=\frac{\mathrm{N} \sigma^{2}}{(\mathrm{~N}-1) \mathrm{D}+\sigma^{2}} \quad \mathrm{D}=\left(\frac{\mathrm{e}}{\mathrm{t}}\right)^{2}
$$


Donde:

t : Valor t de Student con (n-1) grados de libertad y probabilidad (1- $\alpha)$ si es $95 \%$ se puede aproximar a 2.

$\mathrm{e}=$ Margen de error permisible para la estimación de la media.

$\sigma=$ Desviación estándar poblacional.

$\mathrm{N}=$ Número de elementos de la población.

Uno de los problemas para calcular el tamaño de muestra es el desconocimiento del valor de $\sigma$. En la práctica, $\sigma$ puede estimarse a partir de una encuesta anterior de similares características o a partir de una muestra piloto cuyo tamaño suele ser entre 30 y 100 elementos.

Si se requiere la estimación de proporciones, la fórmula anterior es válida con $\sigma^{2}=\mathrm{P}(1-\mathrm{P})$; es decir:

$$
\mathrm{n}=\frac{\mathrm{NP}(1-\mathrm{P})}{(\mathrm{N}-1) \mathrm{D}+\mathrm{P}(1-\mathrm{P})}
$$

En la práctica, no se conoce el valor de $\mathrm{P}$, pues el muestreo tiene como propósito estimar el valor de P. Sin embargo, pueden obtenerse estimaciones preliminares de $\mathrm{P}$ mediante un estudio anterior de similares características o una muestra piloto cuyo tamaño suele ser entre 30 y 100 elementos. En última instancia el valor de $\mathrm{P}$ se puede sustituir por 0.5 y se obtendrá un tamaño de muestra mayor que el requerido, con este valor de $\mathrm{P}$, la varianza poblacional es 0.25 , que resulta ser su máximo valor.

\subsubsection{Software libre para determinación del TEM en muestreo directo simple}

En internet existe una página web de la empresa GMI, que se utiliza para el cálculo de tamaño de muestra para los estudios de investigación de mercado (TEM): <http://es.gmi-mr.com/resources/sample-sizecalculator.php>; en ella se puede determinar fácilmente el tamaño de muestra y el intervalo de confianza. En el gráfico 5 se muestra la ventana para el cálculo: 


\section{Gráfico 5}

Ventana para cálculo de tamaño de muestra en estudios de mercado
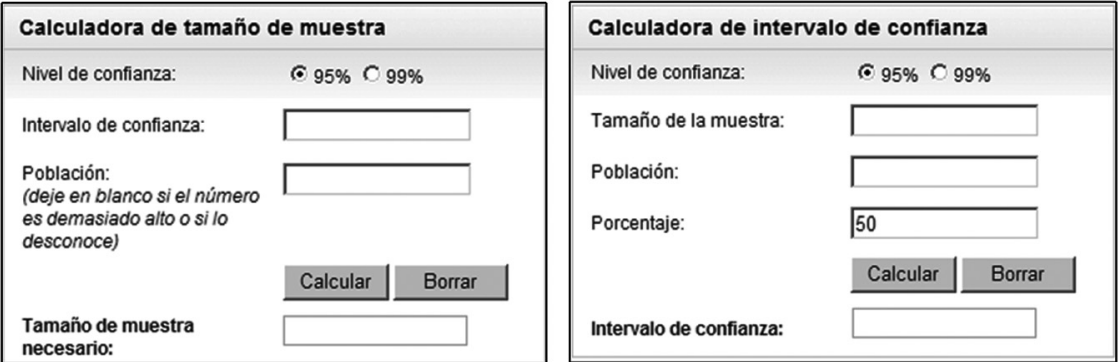

Fuente: <http://es.gmi-mr.com/resources/sample-size-calculator.php>.

\subsection{Tamaño de muestra en el muestreo complejo}

Kish $^{3}$ define un indicador para medir el efecto de un diseño muestral complejo en la estimación de un parámetro, este indicador se define como el cociente de la varianza del diseño muestral complejo $\left(\mathrm{V}_{\text {dis }}\right)$ y la varianza del m.a.s $\left(\mathrm{V}_{\text {mas }}\right)$, es decir: $\mathrm{Ef}=\frac{\mathrm{V}_{\text {dis }}}{\mathrm{V}_{\text {mas }}}$. En aquellas encuestas a gran escala que se basan en muestras complejas el valor $E f$ suele ser mayor que uno debido al grado de complejidad de la muestra. La Encuesta Nacional de Hogares (Enaho) reporta valores de $E f$ entre 1.5 y 3.0 en sus diferentes estimaciones difundidas.

Entonces, al determinar el tamaño de una muestra compleja se debe incorporar el efecto de diseño muestral para asegurar que la varianza de las estimaciones mantengan un nivel de precisión aceptable. Por tanto, el tamaño de muestra de unidades de observación (unidades finales) de una muestra compleja se determina mediante la siguiente fórmula:

$$
\mathrm{n}=\frac{\mathrm{Ef} * \mathrm{~N} \sigma^{2}}{(\mathrm{~N}-1) \mathrm{D}+\mathrm{Ef} * \sigma^{2}} \quad \mathrm{D}=\left(\frac{\mathrm{e}}{\mathrm{t}}\right)^{2}
$$

3 Kish, L. Muestreo de encuestas, 1982. 
Donde:

t : Valor t de Student con (n-1) grados de libertad y probabilidad $(1-\alpha)$ si es $95 \%$ se puede aproximar a 2 .

e $=$ Margen de error permisible para la estimación de la media.

$\sigma=$ Desviación estándar poblacional de unidades finales.

$\mathrm{N}$ = Número de unidades finales de muestreo de la población.

\section{EL MUESTREO DE ACEPTACIÓN}

Es la inspección por muestras en la que se toma la decisión de aceptar o no un producto o servicio. También la metodología que trata de los procedimientos por los que las decisiones de aceptar o no se basan sobre los resultados de la inspección por muestras.

El plan de muestreo de aceptación determina el tamaño de muestra que va a ser utilizada y el criterio asociado de aceptación o rechazo. Protección que proporcionan:

- Dejar pasar un lote que no satisfaga, como si fuera bueno: Riesgo del consumidor $(\beta)$; calculado con la fracción defectuosa tolerable del lote (PDTL) o Nivel de calidad limitante (NCL).

- Rechazar un lote bueno, como si fuera insatisfactorio: Riesgo del productor $(\alpha)$ calculado con el Nivel de calidad aceptable (NCA).

\subsection{Curva operativa}

La curva operativa relaciona la probabilidad de aceptación y su relación con la fracción de defectuosos. Es evidente que cada una ofrece un distinto nivel de protección. Cuando se utiliza un tamaño de muestra fijo o constante, las curvas CO son muy semejantes. Conforme crece el tamaño de la muestra, aumenta la pendiente de la curva. Conforme disminuye el número de aceptación, aumenta la pendiente de la curva.

Minitab nos facilita el cálculo de los planes de muestreo y sus respectivas curvas operativas. A continuación en los gráficos 6,7 y 8 se muestran las ventanas: 


\section{Gráfico 6}

Ventana para los muestreos de aceptación

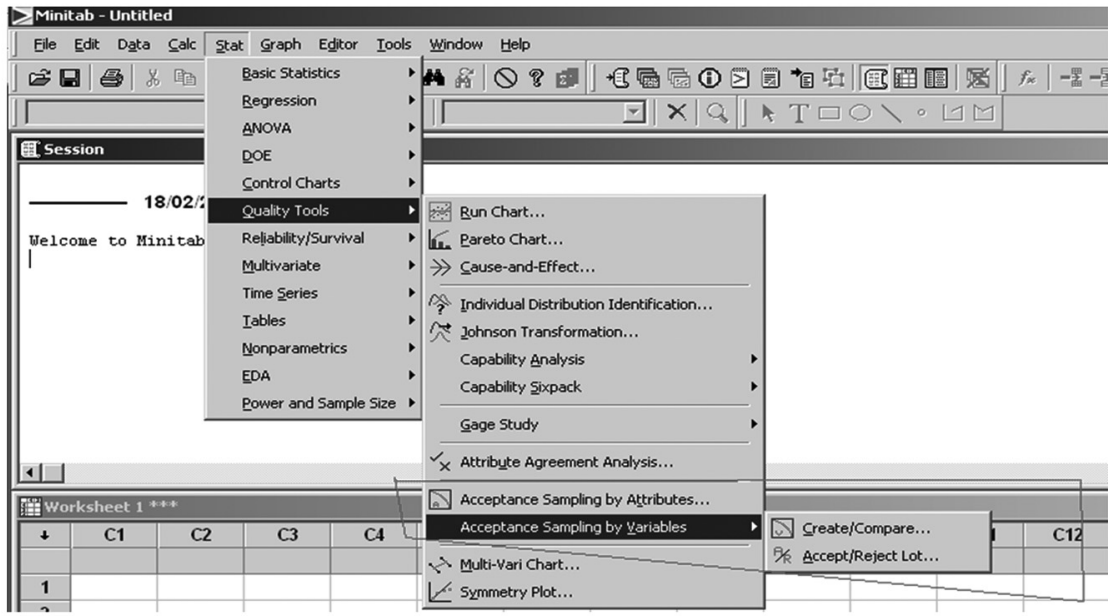

Elaboración propia con uso de Minitab.

Gráfico 7

Ventana para los muestreos de aceptación por atributo

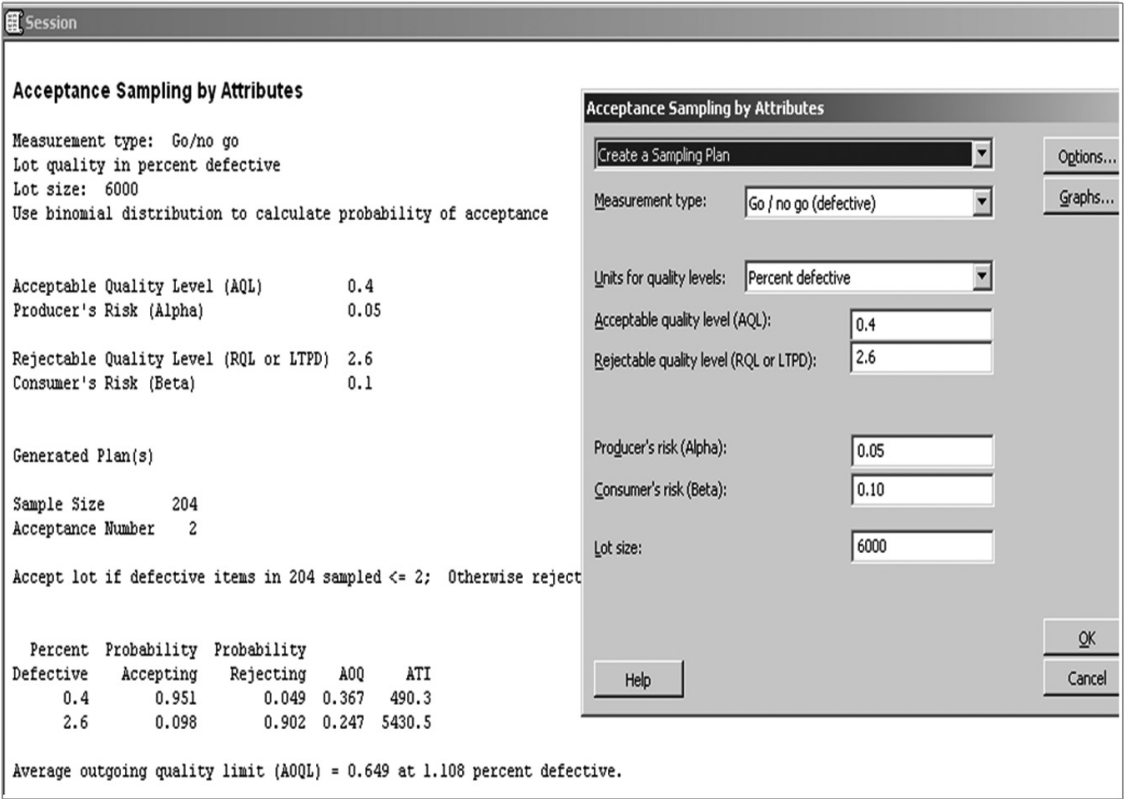

Elaboración propia con uso de Minitab. 


\section{Gráfico 8}

Ventana de las gráficas de curva operativa

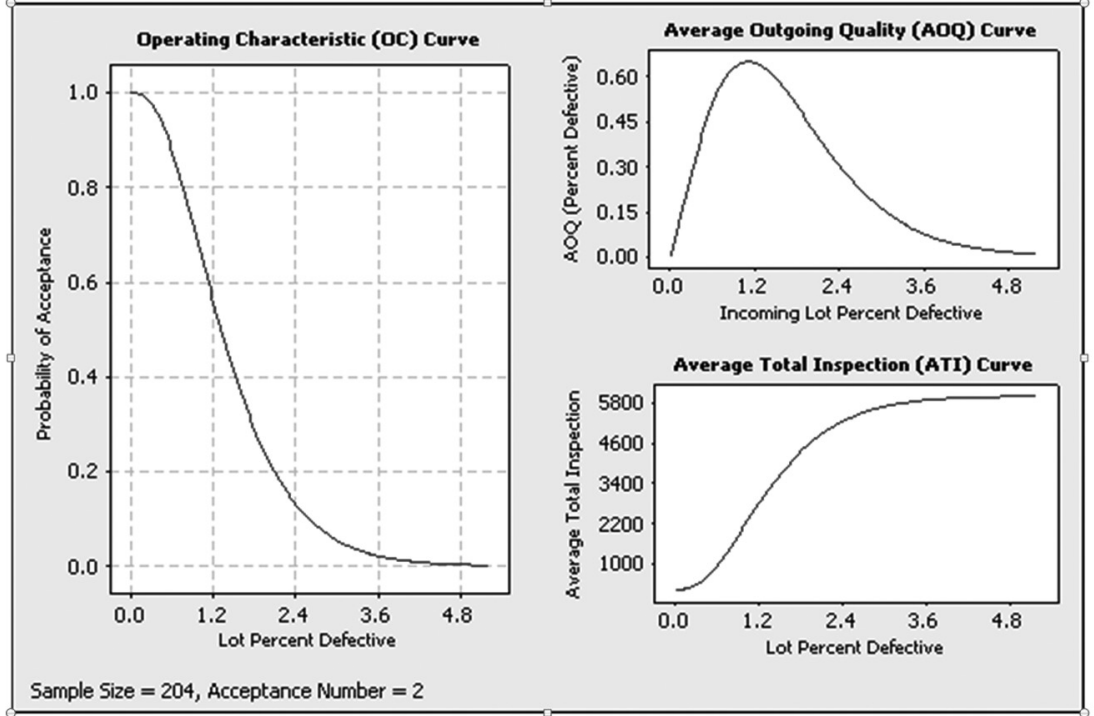

Elaboración propia con uso de Minitab.

\subsection{Tabla Mil.Std 105 E}

La Organización Internacional de Estándares ISO adoptó en 1973 el índice de calidad que usa de manera principal el Mil.Std 105E, que es el nivel de calidad aceptable (NCA), también conocido como AQL (Acceptable Quality Level).

La NTP-ISO 2859-0 1999 es la norma técnica peruana para procedimiento de muestreo para inspección por atributo.

\subsubsection{Diseño de muestreo con la Tabla $105 \mathrm{E}$}

Para diseñar el muestreo con la Tabla 105 E se realiza el procedimiento que se presenta a continuación: 
- Especificar el NCA (AQL).

- Escoger el nivel de inspección, usualmente el nivel II.

- Determinar el tamaño del lote.

- Encontrar la letra de código para el tamaño de la muestra, dependiendo de la tabla A, y de acuerdo con el tamaño del lote.

- Decisión en cuanto al procedimiento de muestreo que se va a utilizar: simple (único), doble, múltiple.

- De acuerdo con la letra, el código, el NCA y el tipo de inspección en la tabla $\mathrm{B}$, se encuentra el plan de muestreo requerido.

- Especificación del NCA

- Debe especificarse en el contrato o por la autoridad responsable.

- Pueden especificarse diferentes NCA para conjuntos de defectos considerados colectivamente o para defectos individuales.

- Los valores de NCA de 10 o menos pueden expresarse en porcentaje defectuoso o en defectos por cada cien unidades; los que superan el 10 deberán ser expresados en defectos por cada cien unidades únicamente.

- Nivel de inspección

- Determina la relación entre el tamaño del lote o conjunto y el tamaño de la muestra.

- El nivel de inspección que deba utilizarse en un caso particular debe ser establecido por la autoridad responsable.

- En la tabla A aparecen tres niveles de inspección - I, II, IIIpara uso general.

- A menos que se especifique otra cosa, se utilizará el nivel II. Sin embargo, el nivel I puede utilizarse cuando se necesite menos distinción, o bien el II para un discernimiento más elevado.

- Los niveles: S-1, S-2, S-3 y S-4 pueden utilizarse donde los tamaños de las muestras necesarias sean relativamente pequeños y los grandes riesgos de muestreo pueden o deben ser tolerados.

- Tipos de muestreo

- Sencillos (único), dobles o múltiples. 
- Además, existen tres tipos de inspección: normal, riguroso y reducido.

- Criterios de cambios de inspección

- Normal: Toda inspección se inicia como normal, y se mantiene así hasta que los siguientes procedimientos de modificación exijan un cambio.

- De normal a rigurosa: cuando dos de cinco lotes consecutivos han sido rechazados.

- De rigurosa a normal: cuando cinco lotes consecutivos se aceptan dentro de la inspección original.

- De reducida a normal: cuando un lote resulta rechazado.

\subsubsection{Uso de la tabla MilStd 105E}

Cuando el estándar se usa para planes porcentuales de artículos defectuosos (disconformes), los NCA van de $0.10 \%$ a $10 \%$. En los planes de defectos (disconformidad) por unidades hay $10 \mathrm{NCA}$ adicionales que llegan hasta 1.000 defectos por 100 unidades. El NCA por lo general está especificado en el contrato o por la autoridad responsable del muestreo.

En la práctica es común elegir el NCA de 1\% para defectos importantes; $2,5 \%$ para defectos menores. Ningún defecto crítico es aceptado. En las páginas 32-33 se presentan las tablas para códigos de letras para determinar el tamaño de muestra del plan de muestreo para atributos y la tabla para definir el plan de muestreo.

Cuando leemos la tabla del cuadro 3 , si el tamaño de muestra y el NCA nos indican una flecha, debemos seguirla y escoger el nuevo plan de muestreo. 
Fernando Kleeberg, Julio César Ramos

\section{Cuadro 3}

Tabla A de letras código para tamaño de muestra Mil. Std 105E

Letras código para el tamaño de las muestras (Mil. Std 105D, tabla I)

\begin{tabular}{|c|c|c|c|c|c|c|c|c|c|}
\hline \multicolumn{3}{|c|}{$\begin{array}{c}\text { Tamaño del lote } \\
\text { o conjunto }\end{array}$} & \multicolumn{4}{|c|}{ Niveles de aceptación } & \multicolumn{3}{|c|}{$\begin{array}{c}\text { Niveles generales } \\
\text { de inspección }\end{array}$} \\
\hline & & & S-1 & S-2 & S-3 & S-4 & I & II & III \\
\hline 2 & a & 8 & $A$ & A & $A$ & $A$ & $A$ & $A$ & B \\
\hline 9 & a & 15 & $A$ & $A$ & $A$ & $A$ & A & $\mathrm{B}$ & $\mathrm{C}$ \\
\hline 16 & $a$ & 25 & A & A & $B$ & B & $B$ & C & $\mathrm{D}$ \\
\hline 26 & a & 50 & $A$ & B & B & $\mathrm{C}$ & $\mathrm{C}$ & $\mathrm{D}$ & $\mathrm{E}$ \\
\hline 51 & a & 90 & $\mathrm{~B}$ & B & $\mathrm{C}$ & $\mathrm{C}$ & $\mathrm{C}$ & $E$ & $\mathrm{~F}$ \\
\hline 91 & a & 150 & $\mathrm{~B}$ & B & $\mathrm{C}$ & $\mathrm{D}$ & $\mathrm{D}$ & $\mathrm{F}$ & G \\
\hline 151 & a & 280 & $\mathrm{~B}$ & $\mathrm{C}$ & $\mathrm{D}$ & $E$ & $E$ & G & $\mathrm{H}$ \\
\hline 281 & a & 500 & B & C & $\mathrm{D}$ & $E$ & $\mathrm{~F}$ & $\mathrm{H}$ & $\mathrm{J}$ \\
\hline 501 & a & 1200 & $\mathrm{C}$ & C & $E$ & $\mathrm{~F}$ & G & $\mathrm{J}$ & $\mathrm{K}$ \\
\hline 1201 & $a$ & 3200 & C & $\mathrm{D}$ & $E$ & G & $\mathrm{H}$ & $\mathrm{K}$ & $\mathrm{L}$ \\
\hline 3201 & a & 10000 & C & $D$ & $\mathrm{~F}$ & G & $\mathrm{J}$ & $\mathrm{L}$ & $\mathrm{M}$ \\
\hline 10001 & a & 35000 & $\mathrm{C}$ & $\mathrm{D}$ & $\mathrm{F}$ & $\mathrm{H}$ & $\mathrm{K}$ & $M$ & $\mathrm{~N}$ \\
\hline 35001 & a & 150000 & $\mathrm{D}$ & $E$ & G & $\mathrm{J}$ & $\mathrm{L}$ & $\mathrm{N}$ & $\mathrm{P}$ \\
\hline 150001 & $a$ & 500000 & $\mathrm{D}$ & $E$ & G & $\mathrm{J}$ & M & $\mathrm{P}$ & Q \\
\hline 500001 & $y$ & más & $\mathrm{D}$ & $E$ & $\mathrm{H}$ & $\mathrm{K}$ & $\mathrm{N}$ & Q & $\mathrm{R}$ \\
\hline
\end{tabular}

Fuente: <http://www.sqconline.com/>.

A continuación se presenta la tabla II-A de la Mil.std 105 para inspección por atributos de muestreo único: 


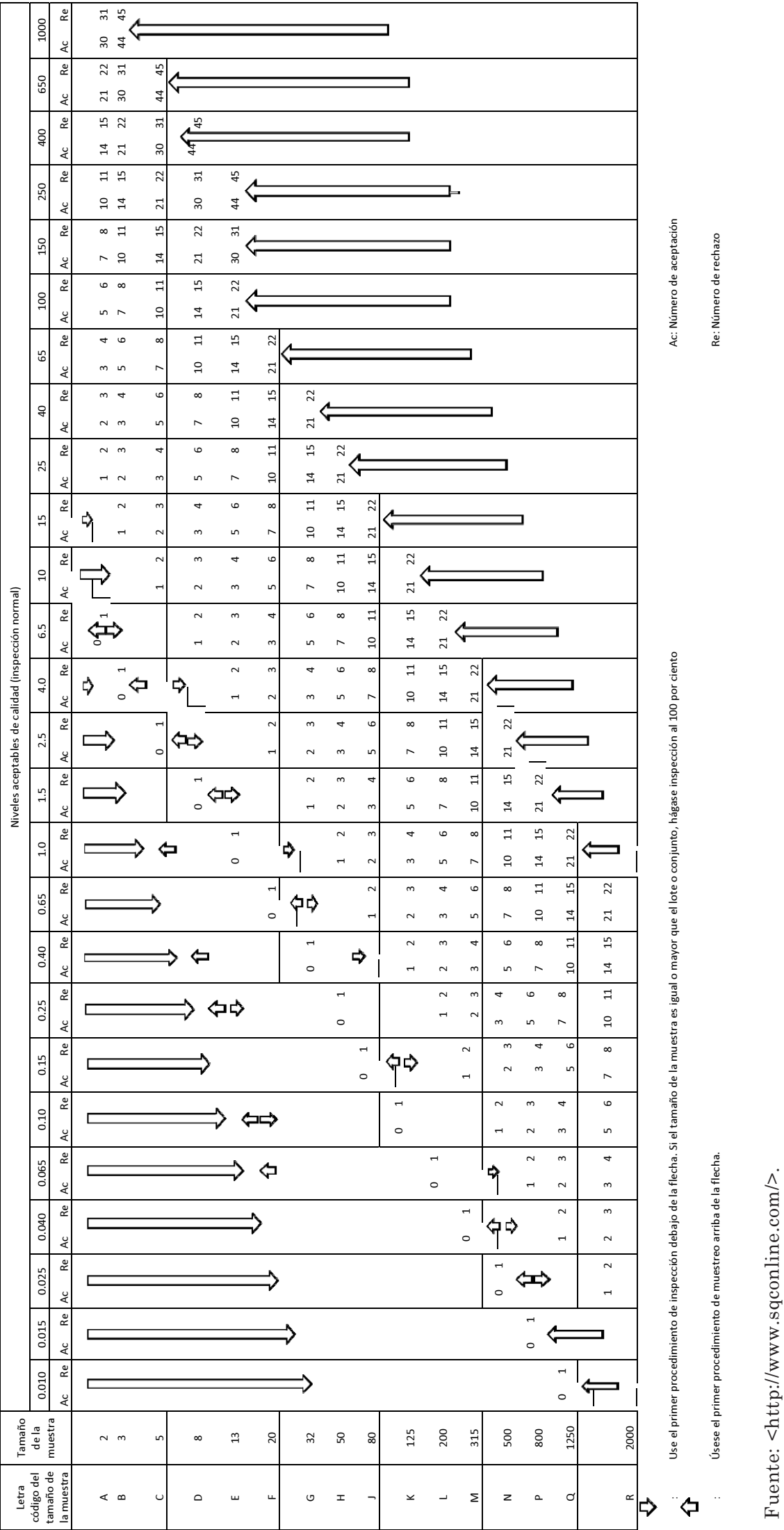


El SQC online es un software libre del uso de las tablas Mil.Std $105 \mathrm{E}$ y 414 para calcular los tamaños de muestra y número de aceptación a partir del tamaño de lote y el nivel de calidad aceptable, tanto para muestreo por atributo como de datos variables (véase el cuadro 4).

\section{Cuadro 4}

Ventana de tabla a utilizar

\begin{tabular}{|c|l|l|}
\hline Table & What is it for? & $\begin{array}{l}\text { Civilian } \\
\text { Counterpart }\end{array}$ \\
\hline Military Standard 105E & Sampling plans for attribute data & $\begin{array}{l}\text { ANSVASQC Z1.4 } \\
\text { ISO 2859, BS6001 }\end{array}$ \\
\hline Military Standard 414 & Sampling plans for measurement data & $\begin{array}{l}\text { ANSVASQC Z1.9 } \\
\text { ISO } 3951, \text { BS6002 }\end{array}$ \\
\hline Military Standard 1235C & $\begin{array}{l}\text { Sampling inspection plans for } \\
\text { continuous production, Procedure } \\
\text { CSP-1 }\end{array}$ & \\
\hline MIL.HDBK-217 & $\begin{array}{l}\text { Calculation of MTBF and failure rates } \\
\text { for electrical and electronic } \\
\text { comnonents. devices. and eauinment. }\end{array}$ & \\
\hline
\end{tabular}

Fuente: <http://www.sqconline.com/>.

En los cuadros 5 y 6 se presenta la ventana en el SQC online para el muestreo por atributo; se solicita el tamaño de lote, el NCA, el nivel de inspección y el tipo de inspección; luego se somete y nos muestra el plan de muestreo que se debe realizar (tamaño de muestra y el número de aceptación). El software también nos muestra la curva operativa de plan de muestreo.

\section{Cuadro 5}

Ventana del muestreo por atributo del software SQC online

\begin{tabular}{|l|l|}
\hline Enter your process parameters: \\
\hline Batch size (N): & 3201 to 10000 \\
\hline AQL: & $0.40 \%$ \\
\hline Inspection Level: & Nor \\
\hline Type of inspection: & Normal \\
\hline Submit \\
\hline
\end{tabular}

Fuente: $<$ http://www.sqconline.com/>. 


\title{
Cuadro 6
}

Ventana del muestreo por atributo del software SQC online

\author{
Sampling by Attributes \\ Militany Standard 105E (ANSI/ASQC Z1.4, ISO 2859) Tables \\ For a lot of 3201 to 10000 items, and $\mathrm{AQL}=\mathbf{0 . 4 \%}$, normal \\ inspection plans are:
}

The Single sampling procedure is:

Sample 200* items.

If the number of non-conforming items is

2 or less --> accept the lot.

3 or more $->>$ reject the lot.

The Double sampling procedure is:

\begin{tabular}{|l|l|}
\hline Step 1: & $\begin{array}{l}\text { Sample } 125^{\star} \text { items. } \\
\text { If the number of non-conforming items is } \\
\text { 0 --> accept the lot. } \\
\text { 3 or more }-->\text { reject the lot. } \\
\text { Otherwise, continue to step 2. }\end{array}$ \\
\hline Step 2: & $\begin{array}{l}\text { Sample } 125^{\star} \text { additional items. } \\
\text { If the total number of non-conforming items is } \\
\text { 3 or less --> accept the lot. } \\
\text { 4 or more }-->\text { reject the lot. }\end{array}$ \\
\hline
\end{tabular}

Fuente: http://www.sqconline.com/.

\subsection{Tabla Mil.Std 414}

La norma del Departamento de la Defensa de Estados Unidos para inspección de muestreo de aceptación por variables es llamada Military Standard 414 (ISO Std. 3951) (Sampling Procedures and Tables for Inspection by Variables for Percent Defective), emitida en 1957 para ser utilizada como alternativa a la norma de atributos Mil Std. 105A expedida en 1950; existen tablas que trabajan con el sigma conocido y otras con sigma desconocido.

Procedimiento de uso: Tabla Mil.Std 414 con sigma conocido o desconocido: 
- El tamaño del lote.

- Tipo de muestreo sencillo.

- El tamaño de la muestra.

- El nivel de calidad aceptable (Inspección Normal o Severa).

- La constante de aceptabilidad (k).

- En el caso de sigma desconocido se calculará el sigma con los valores de la muestra

- El tamaño de muestra con la letra de código de la tabla A2-414 mostrada en el cuadro 7.

- Xbarra es el promedio de los datos, LIE (límite inferior de la especificación).

- El valor de Z.

- $\mathrm{Z}=($ Xbarra $-\mathrm{LIE}) / \mathrm{s}$; Si $\mathrm{Z}<\mathrm{k}$ Se rechaza el lote.

Si $Z \geq k \quad$ Se acepta el lote.

Se supone una distribución básica normal, si no los riesgos de utilizar la tabla serán mayores. En la tabla A2414 mostrada en el cuadro 7 existen cinco niveles generales de inspección, siendo el IV el considerado como normal.

En el cuadro 8 se presenta la tabla Milstd 414, donde se observa que las variables estándar se clasifican para los mismos NCA que los atributos estándar dentro los intervalos de 0.04 a $15 \%$, pero las clases de tamaño son diferentes. La tabla maestra para inspección normal y severa puede ser para procesos de variabilidad conocida como desconocida: 


\section{Cuadro 7}

Tabla A para definir la letra código del tamaño de muestra

Tabla A.2 MIL STD 414

Letras código de tamaño muestral

\begin{tabular}{lccccc}
\hline Tamaño del lote & I & II & III & IV & V \\
\hline 3 a 8 & B & B & B & B & C \\
9 a 15 & B & B & B & B & D \\
15 a 25 & B & B & B & C & E \\
26 a 40 & B & B & B & D & F \\
41 a 65 & B & B & C & E & G \\
66 a 110 & B & B & D & F & H \\
111 a 180 & B & C & E & G & I \\
181 a 300 & B & D & F & H & J \\
301 a 500 & C & E & G & I & K \\
501 a 800 & D & F & H & J & L \\
801 a 1,300 & E & G & I & K & L \\
1,301 a 3,200 & F & H & J & L & M \\
3,201 a 8,000 & G & I & L & M & N \\
8,001 a 22,000 & H & J & M & N & O \\
22,001 a 110,000 & I & K & N & O & P \\
110,000 a 550,000 & I & K & O & P & Q \\
550,000 y mayor & I & K & P & Q & Q \\
& & & & & \\
\hline
\end{tabular}

Fuente: http://www.sqconline.com/. 


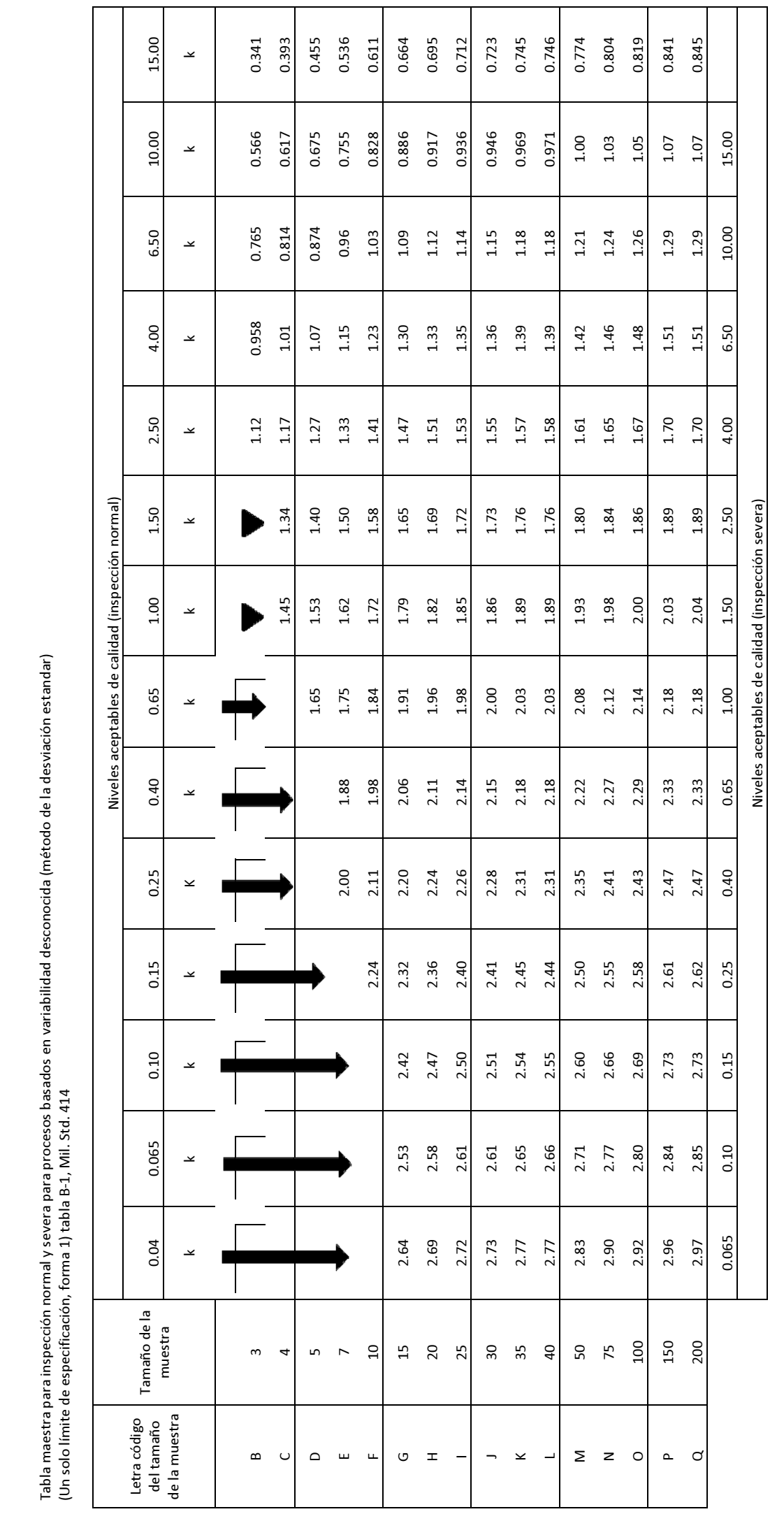


En el cuadro 9 se presenta la ventana en el SQC online para el muestreo para datos variables; se solicita el tamaño de lote, el NCA, el nivel de inspección y el tipo de inspección; luego se somete y nos presenta el plan de muestreo que se debe realizar (tamaño de muestra y el número de aceptación). También el software nos muestra la curva operativa del plan de muestreo.

\section{Cuadro 9}

Ventana de ingreso de parámetros y ventana para estimar el porcentaje de defectuosos

\begin{tabular}{|l|l|}
\hline \multicolumn{2}{|l|}{ Enter your process parameters: } \\
\hline Variability & $\begin{array}{l}\text { ○ Unknown } \\
\text { Known }\end{array}$ \\
\hline Batch/lot size (N): & 1301 to $3200 \quad$ \\
\hline AQL: & $0.40 \%$ \\
\hline Inspection Level: & IV \\
\hline Type of inspection: & Normal \\
\hline Submit & \\
\hline
\end{tabular}

Sampling by Variables

\section{Military Standard 414 (ANSI/ASQC Z1.9) Tables}

For a lot of 1301 to 3200 items, and $A Q L=0.40 \%$, with inspection level IV , the Normal inspection plan is:

Sample $40^{*}$ items.

If the estimated percent of non-conforming (defective) items is $1.26 \%$ or less $\rightarrow>$ accept the lot.

Otherwise, reject it.

To estimate the percent of non-conforming items in your process, take a sample of size $\mathbf{4 0}$ and enter the values into the next table.

\begin{tabular}{|c|l|l|}
\hline \multicolumn{2}{|c|}{ To estimate your process \% non-conforming (defectives ) enter: } \\
\hline Sample Average $(\overline{\mathbf{x}}):$ & & The average of the 40 measurements \\
\hline $\begin{array}{c}\text { Process Standard } \\
\text { Deviation (s): }\end{array}$ & The standard deviation of the 40 measurements \\
\hline $\begin{array}{c}\text { Lower Specification } \\
\text { Limit: }\end{array}$ & $\begin{array}{l}\text { The smallest value for your measurement that is } \\
\text { considered acceptable. } \\
\text { Leave blank if there is no lower limit. }\end{array}$ \\
\hline $\begin{array}{c}\text { Upper Specification } \\
\text { Limit: }\end{array}$ & $\begin{array}{l}\text { The largest value for your measurement that is } \\
\text { considered acceptable. } \\
\text { Leave blank if there is no upper limit. }\end{array}$ \\
\hline \multicolumn{3}{|c|}{} \\
\hline \multicolumn{2}{|c|}{ Submit } \\
\hline
\end{tabular}

Fuente: <http://www.sqconline.com>. 
Como se observa el sqconline nos guía para definir nuestro plan y aceptar o rechazar el lote según el porcentaje defectuoso sea mayor o menor al k límite de aceptación (el k límite es un $\mathrm{Z}$ límite de aceptación que puede ser leído en la tabla normal en términos de porcentaje [\%] límite de defectuosos para aceptación).

\section{BIBLIOGRAFÍA}

Bhattacharyya, Gouri K. y Richard A. Johnson. Statistical concepts and methods. Nueva York: John Whiley and Sons, Inc., 1977.

Casal, J. y E. Mateu. "Tipos de Muestreo". Revista de Epidemiología y Medicina Preventiva 1. Barcelona: Universitat Autónoma de Barcelona, 2003.

Cochran, W. Técnicas de muestreo. México: Cecsa, 1980.

GMI. Calculadora de tamaño de muestra [en línea]. <http://es.gmi-mr. com/resources/sample-size-calculator.php>.

Kish, L. Muestreo de encuestas. México: Trillas, 1982.

Minitab Inc. International Sales and Support Quality Minitab 15. Software estadístico para control de calidad. Estados Unidos, 2008.

Montgomery, Douglas. Control estadístico de la calidad. 3. ${ }^{a}$ edición. México, D.F.: Limusa Wiley, 2005.

Pérez, César. Técnicas de muestreo estadístico: Teoría, práctica y aplicaciones. México, D.F.: Alfaomega, 2000.

Raj, D. Teoría del muestreo. México: Fondo de Cultura Económica, 1984.

Rubio, Arturo. "Técnicas de Muestreo". Maestría en estadística aplicada. Lima: Universidad Nacional Agraria La Molina, 2005.

Ruiz Muñoz, D. Manual de estadística. España: Universidad Pablo de Olavide. Eumed-Net, 2008.

Scheaffer, Mendenhall, Ott. Elementos de muestreo. México: Iberoamérica, 1987.

SQConline. Software libre para el control de calidad y control estadístico de procesos [en línea] <http://www.sqconline.com/>. 